\title{
Perceiving shape from profiles
}

\author{
FRANK E. POLLICK \\ ATR Human Information Processing Research Laboratories, Kyoto, Japan
}

\begin{abstract}
Four experiments related human perception of shape from profiles to current theoretical predictions. In Experiment 1, judgments of structure and motion were obtained for single- and dualellipsoid displays rotating about various axes. Ratings were highest when the axis of rotation was in the image plane and were influenced by the number of ellipsoids and the orientation of a single ellipsoid. The subsequent experiments explored the effect of orientation on shape judgments of a single ellipsoid. The results of Experiments 2 and 3 suggested that the effect of orientation found in Experiment 1 was not due to either the inability of certain orientations to be perceived as three-dimensional objects or to two-dimensional artifacts. It was thus argued that this effect of orientation was due to points of correspondence in relative motion that arise when the major axis is not perpendicular to the axis of rotation. In Experiment 4, subjects provided judgments of both shape and angular velocity. The elevated ellipsoids that were judged as larger were also judged as rotating more slowly. The inverse relationship between size and angular velocity is consistent with current theories. The connection between theory and data was further demonstrated by applying a shape-recovery algorithm to the stimuli used in Experiment 4 and finding a similar tradeoff between angular velocity and shape.
\end{abstract}

The single profile (silhouette) of a solid object provides much information about the shape of the object, and thus it is reasonable to assume that a series of profiles of a moving object should provide even more information. However, once the object begins to move, the set of points on the solid object that create the profile slide across the surface of the object. Because of this sliding, few points on the profile correspond to the same three-dimensional (3-D) location as the object moves. This has usually been seen as a detriment for computational theories, and contrasting evidence has shown that, for humans, the profiles of an object in motion are sufficient to produce a compelling percept of a solid object moving in depth (Cortese \& Andersen, 1991; Norman, Todd, \& Fukuda, 1992; Pollick, 1989; Todd, 1985; Wallach \& O'Connell, 1953). However, recent theories of the recovery of 3-D form from profiles (Cipolla \& Blake, 1992; Giblin \& Weiss, 1987; Vaillant \& Faugeras, 1992) have shown that if an object's global motion is known, this lack of correspondence is not a problem, and the motion of noncorresponding points can yield exact information about properties of local surface shape. This paper looks at the perception of shape from profiles in the perspective of these current computational theories.

The support of the Office of Naval Research (Contract N00014-88$\mathrm{K}-0354)$ is gratefully acknowledged. Portions of these experiments and simulations were performed at the University of California, Irvine. For their helpful comments, I thank Jack Beusmans, David Eby, and Peter Giblin. Correspondence should be addressed to F. Pollick, ATR Human Information Processing Research Laboratories, 2-2 Hikaridai, Seikacho, Soraku-gun, Kyoto 619-02, Japan (e-mail: fpollick@hip.atr.co.jp).

-Accepted by previous editor, Charles W. Eriksen
The theories of 3-D shape recovery developed by Giblin and Weiss (1987), Cipolla and Blake (1992), and Vaillant and Faugeras (1992) provide techniques to compute a depth map and local surface curvature from the profiles of a smooth surface in motion. The work of Giblin and Soares (1988) extended the analysis to singularities of the profile. The model of Giblin and Weiss assumed a series of orthographic projections with coplanar viewing directions (and a generalization of the Giblin and Weiss model can accommodate noncoplanar viewing directions; see Pollick, Giblin, Rycroft, \& Wilson, 1992). Cipolla and Blake, as well as Vaillant and Faugeras, considered the more general case of perspective projection and arbitrary motion of the viewing direction, and the robustness of such algorithms has been demonstrated by Blake and Cipolla (1991).

All of the above-mentioned theories assumed that the relative motion between the viewing direction and the object was known. This assumption is problematic for modeling human vision, and thus recent theoretical research has addressed what can be recovered about the motion from measurements of the profiles themselves (Giblin, Pollick, \& Rycroft, 1993; Giblin, Rycroft \& Pollick, in press). Results of this research have shown that, by assuming constant angular velocity motion over an arbitrary interval of time, it is possible to uniquely recover the tilt of the axis of rotation and to specify the relationship between possible values of the angular velocity and slant of the axis of rotation. The results are similar to those from the recovery of structure from two orthographic views (Bennett, Hoffman, Nicola, \& Prakash, 1989; Huang \& Lee, 1989; Koenderink \& Van Doorn, 1991; Todd \& Bressan, 1990). 
Without knowledge of the rate at which the viewing direction is moving relative to an object, it is impossible to uniquely recover the object's 3-D shape. This situation is most clearly demonstrated if the axis of rotation is perpendicular to the viewing direction, when a simple inverse relationship holds between shape and angular velocity. This relationship is illustrated in Figure 1 and discussed in detail in the Appendix using the model of Giblin and Weiss (1987). If angular velocity is unknown, there are an infinite number of possible shape interpretations. (Two of these interpretations are shown in Figure 1.) The possible interpretations are long objects rotating slowly through a small angle or short objects rotating quickly through a large angle. Unique shape interpretations are available only if angular velocity is known. Thus, one would predict that any errors in the perception of angular velocity would make themselves apparent as errors in the perception of shape (Pollick, 1989).

The question then naturally arises, under what conditions is it possible to recover angular velocity from the profiles of a rotating object? The possibility explored in the current research is that this information could be provided by the relative motion of points of correspondence, which are those identifiable points in the image that always project back to the same location on the 3-D object. For two successive profiles of a solid object, regardless of the motion, there is at least one pair of corresponding points (Giblin et al., in press; Rieger, 1986). However, only when the axis of rotation is perpendicular to the viewing direction do the same points remain in correspondence over an entire rotation sequence and trace out straight lines in the direction perpendicular to the axis of rotation. Some of the different types of correspondence that can exist on profiles are illustrated in Figure 2.

When the points of correspondence follow straight paths, it is possible to recover the angular velocity. For

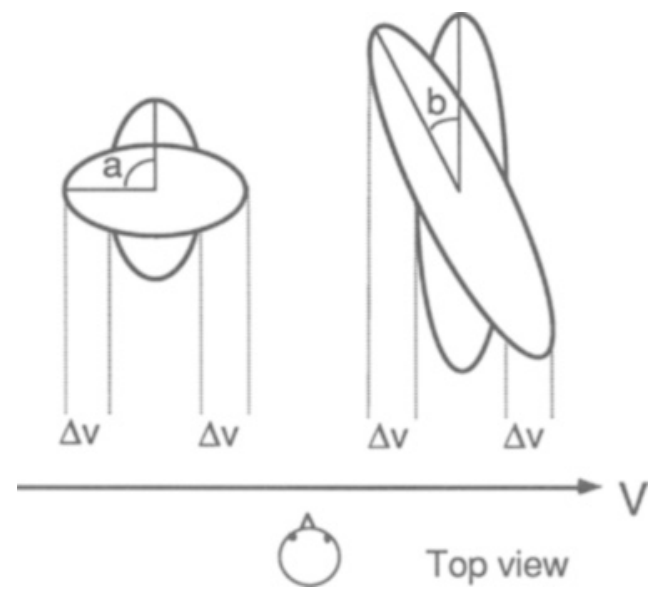

Figure 1. Given the identical change $\Delta v$ in the image, there are an infinite number of possible interpretations of the shape. Two of these are shown: a rounder object rotating through an angle $a$ or a narrow object rotating through an angle $b$.
A

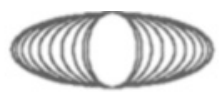

B
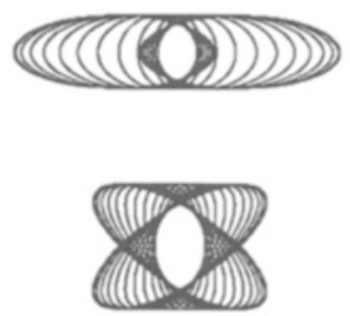

C D
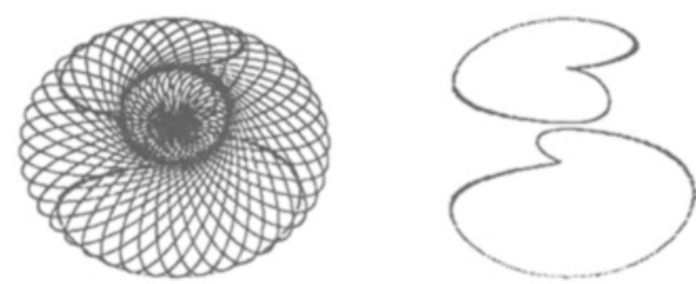

Figure 2. Families of profiles for a complete rotation, and the resulting trace of the projection of the points of correspondence (left, family of profiles and trace; right, trace alone). When the axis of rotation is perpendicular to the line of sight, there can be one of the following: (A) no motion of the points of correspondence (vertical axis through the center of the ellipsoid, (B) absolute motion of the points of correspondence (ellipsoid translated away from the vertical axis), and (C) relative motion of the points of correspondence (same vertical axis of rotation but the ellipsoid is first rotated about the line of sight). When the axis is not perpendicular to the line of sight there is not correspondence in the normal sense, but one can instantaneously identify points of the profile (D) that correspond to the intersection of consecutive critical sets (contour generators) on the surface. These points on the surface are known as frontier points (Giblin, Pollick, \& Rycroft, 1993). Frontier points are similar to true points of correspondence because their image velocity in the direction normal to the profile (the component in the direction of the projection of the axis of rotation) is identical to the projection of the true three-dimensional velocity of the frontier point.

example, if we consider the ellipsoid in Figure $2 \mathrm{C}$ and fixate on one of the points of correspondence, the relative motion of the other point can be used to recover the structure by using a structure-from-motion analysis that assumes fixed-axis rotation (Hoffman \& Bennett, 1985). It should be noted that points of correspondence on profiles do not necessarily need to move relative to one another (consider Figures 2A and 2B), but relative motion would enable angular velocity to be computed in an object-centered coordinate system.

To perform the calculation of angular velocity from points of correspondence, it would of course be necessary to have identified the points of correspondence and marked their trajectories. Computational studies show that, for profiles, when the axis is perpendicular to the viewing 
direction, the points of correspondence can be reliably recovered (Giblin et al., 1993). Evidence from human psychophysics parallels this finding, indicating that, for profiles (as well as for other structure-from-motion displays), subjects are best at recovering the direction of the axis of rotation when it is perpendicular to the viewing direction (Pollick, Nishida, Koike, \& Kawato, 1993).

The notion of points of correspondence in relative motion can be used to interpret the results of Todd (1985). Todd demonstrated that subjects report a compelling perception of 3-D shape from the profiles of two ellipsoids of revolution joined together. (The major axes of the two ellipsoids were orthogonal, and their motion was rotation about a vertical axis.) He also investigated the case of a single zero-elevation ellipsoid rotating about a vertical axis and found that subjects reported a much less compelling percept of 3-D shape. This result of Todd is consistent with the idea that points of correspondence in relative motion provide a cue to motion-in-depth. For the two ellipsoids, there were two pairs of points of correspondence, one pair for each ellipsoid. As the ellipsoids rotated, these pairs moved relative to one another, just as the points of correspondence move for a single elevated ellipsoid. This is in contrast with the motion of corresponding points for the single zero-elevation ellipsoid for which there was no movement between the points of correspondence. Thus, the difference Todd found between a single ellipsoid and two ellipsoids joined together can be explained by the existence of points of correspondence in relative motion.

Both theory and empirical results suggest that perceived angular velocity and relative motion of points of correspondence could influence the perception of shape from profiles. The present experiments explored the influence of points of correspondence and whether judgments of shape were inversely related to judgments of angular velocity. In Experiment 1, to support the hypotheses that points of correspondence in relative motion contribute to the perception of shape from profiles, qualitative judgments of shape and motion were obtained from the profiles of various configurations of rotating ellipsoids. The results of this experiment indicated that displays with points of correspondence in relative motion were more compelling in producing the perception of motion in depth than were displays with stationary points of correspondence. Thus, Experiments 2 and 3 attempted to quantify more precisely the reports of perceived shape and to confirm that reports of greater motion in depth were not caused by either (1) a total failure to perceive 3-D shape in profile displays with stationary points of correspondence (Experiment 2) or (2) 2-D artifacts in the displays with points of correspondence in relative motion (Experiment 3). Having found that these two factors could not entirely account for the effect found in Experiment 1, Experiment 4 explored whether or not the perception of greater motion depth was associated with a decrease in perceived angular velocity, as theory would predict. Finally, a computer simulation of the distance relationship of Giblin and Weiss (1987) was performed to compare its output with the psychophysical results of Experiment 4.

\section{EXPERIMENT 1}

The purpose of Experiment 1 was to explore whether, over a broad range of stimuli, the notion of points of correspondence in relative motion could be used to interpret the perception of shape from profiles. To achieve this end, subjects rated their impressions of rigidity and motion in depth for a variety of profile displays containing either one or two ellipsoids rotating about a fixed axis of rotation. The orientation of the ellipsoid(s) and the axis of rotation were combined so that different motions of the points of correspondence were obtained for the different combinations. For the displays with axes of rotation perpendicular to the viewing direction, these combinations included ellipsoids with (1) no motion of the points of correspondence (Figure 2A) (2) absolute, but not relative, motion of the points of correspondence (Figure 2B), or (3) relative motion of the points of correspondence (Figure $2 \mathrm{C}$ ). For the displays with axes of rotation nonperpendicular to the viewing direction, regardless of the number of ellipsoids, there was no correspondence in the normal sense, although it would have been possible to locate and track frontier points (Figure 2D).

\section{Method}

Subjects. Five staff members with normal or corrected vision volunteered for the experiment. All of the subjects were naive to the purpose of the experiment.

Stimuli and Apparatus. A stimulus simulated an opaque object moving in front of a background plane of randomly placed dots (Cortese \& Andersen, 1991). The motion simulated by the opaque object was constant angular velocity rotation about a fixed axis and was displayed on the screen of a Masscomp 5800 computer $(1,152 \times 910$ resolution). There was a total of 24 stimuli, produced from each of the eight configurations described in Table 1 , rotating about one of three axes of rotation $[(0,1,0),(1,1,1),(3,1,2)$, in which $(0,0,1)$ would be the viewing direction and $(0,1,0)$ the vertical]. Displays were viewed monocularly through a reduction tube that limited the field of view to $6^{\circ}$ (viewing distance approximately $2 \mathrm{~m}$ ). The visual arc subtended by the standard object at its maximum 2-D extent was $2^{\circ}$. Forty-six frames were needed to display one complete rotation, and they were displayed with an SOA of $150 \mathrm{msec}$.

Table 1

Ellipsoid Parameters for Experiment 1

\begin{tabular}{ccccccccc}
\hline Display & Ellipsoid(s) & A & B & C & Elevation & $d 1$ & $d 2$ & $d 3$ \\
\hline 1 & standard & 1.2 & 0.6 & 0.6 & 0.0 & 0.0 & 0.0 & 0.0 \\
2 & standard & 1.2 & 0.6 & 0.6 & 0.0 & 0.7 & 0.2 & 0.2 \\
3 & standard & 1.2 & 0.6 & 0.6 & 30.0 & 0.0 & 0.0 & 0.0 \\
4 & standard & 1.2 & 0.6 & 0.6 & 0.0 & 0.0 & 0.0 & 0.0 \\
& standard & 1.2 & 0.6 & 0.6 & 30.0 & 0.7 & 0.2 & 0.2 \\
5 & standard & 1.2 & 0.6 & 0.6 & 0.0 & 0.0 & 0.0 & 0.0 \\
& pancake & 1.2 & 1.0 & 0.3 & 30.0 & 0.7 & 0.2 & 0.2 \\
6 & standard & 1.2 & 0.6 & 0.6 & 0.0 & 0.0 & 0.0 & 0.0 \\
& round & 0.9 & 0.7 & 0.6 & 30.0 & 0.7 & 0.2 & 0.2 \\
7 & standard & 1.2 & 0.6 & 0.6 & 0.0 & 0.0 & 0.0 & 0.0 \\
& long-thin & 1.0 & 0.2 & 0.4 & 30.0 & 0.7 & 0.2 & 0.2 \\
8 & standard & 1.2 & 0.6 & 0.6 & 0.0 & 0.0 & 0.0 & 0.0 \\
& long-thin & 1.0 & 0.2 & 0.4 & 0.0 & 0.7 & 0.2 & 0.2 \\
\hline
\end{tabular}

Note-Initial positions of the displays were constructed by taking the ellipsoid $x^{2} / A^{2}+y^{2} / B^{2}+z^{2} / C^{2}=1$ and first rotating it about the $z$-axis by the angle elevation and then translating it along the vector $(d 1, d 2, d 3)$. 
Procedure. Each subject participated in a single session that consisted of $\mathbf{4 8}$ trials. The $\mathbf{4 8}$ trials were divided into two blocks, with the first block for practice. The 24 stimuli were randomly ordered in each of the blocks. The subjects were instructed to give rating judgments about the structure and motion of each stimuli. The structure judgment involved whether or not the object appeared as a rigid 3-D object. The ratings corresponded to the following judgments: 1 for no perceived 3-D rigidity; 3 for sometimes rigid, sometimes nonrigid; and 5 for always rigid in 3-D. The motion judgments were intended to show whether or not the object was being perceived as moving in depth. Judgments of 1 corresponded to motion that was always in the image plane; 3 corresponded to motion sometimes in the image plane, sometimes in depth; and 5 corresponded to motion always in 3-D. The subjects had unlimited time to make their responses since stimuli were rotated continuously until a response was made.

\section{Results and Discussion}

The subjects' judgments of the motion and rigidity of the ellipsoids were analyzed by an analysis of variance (ANOVA). The factors used in the ANOVA were type of display and axis of rotation. For the shape judgments, there was a significant effect of axis of rotation $[F(2,8)=$ $20.4, p<.05]$. For the motion judgments, there was a significant effect of both axis of rotation $[F(2,8)=9.9$, $p<.05]$ and type of display $[F(7,28)=6.3, p<.05]$. The trends of the results for both the shape and motion responses are shown in Figure 3.

The most striking results shown in Figure 3 are the higher overall ratings when the axis of rotation was perpendicular to the line of sight and when the display comprised multiple ellipsoids. Looking closely at the results for axis of rotation perpendicular to the line of sight reveals that ratings were lowest for single ellipsoids without points of correspondence in relative motion; they increased for single ellipsoids with points of correspondence in relative motion and increased further when more points of correspondence were introduced by the second ellipsoid. This result is identical to that reported by Todd (1985), and it has to be noted that, as Norman et al. (1992) have suggested, the increase in performance with dual ellipsoids might also have been due to the addition of information at the boundary of the intersection of the ellipsoids.

\section{EXPERIMENT 2}

One possible explanation for the elevation effect of Experiment 1 is that, for displays without points of correspondence in relative motion, subjects cannot organize the display into a 3-D percept. Thus, Experiment 2 explored the subjects' ability to judge a property of the 3-D shape of zero-elevation ellipsoids of revolution. The subjects were presented with ellipsoids of various eccentricities (eccentricity is defined as the ratio of major to minor axis of the ellipsoid of revolution) and were asked to match their cross-sectional shape with an ellipse of adjustable eccentricity.

\section{Method}

Subjects. Six naive undergraduate students volunteered for the experiment and received extra course credit for their participation.
A
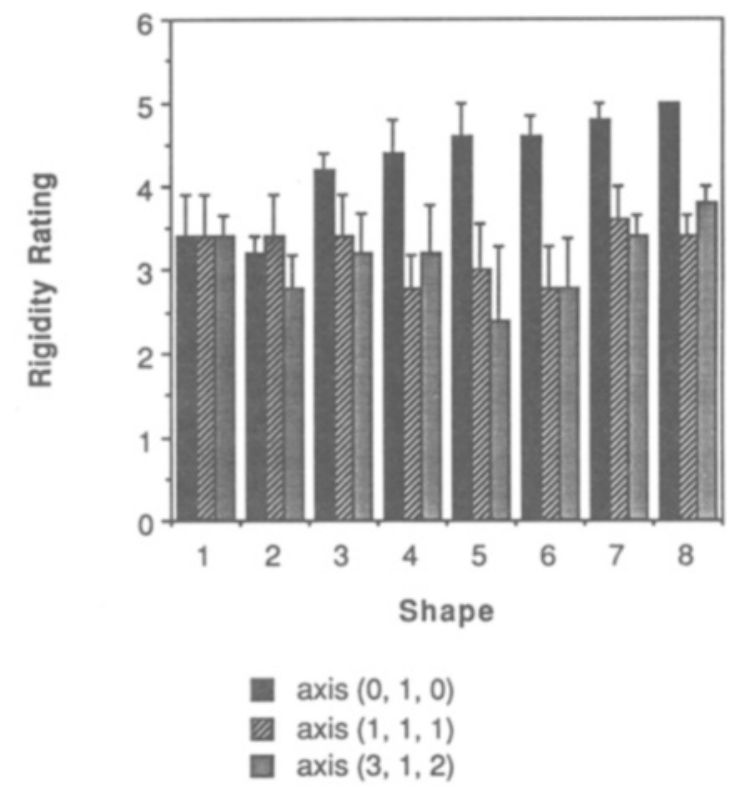

B

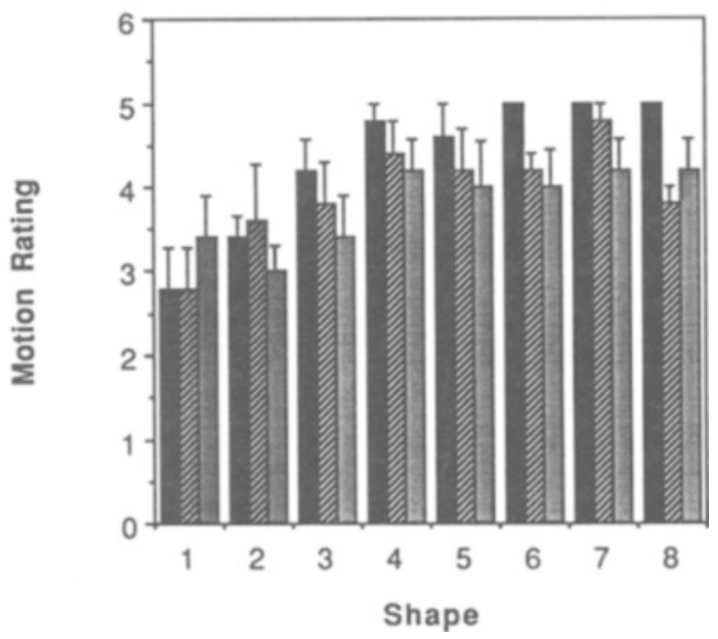

Figure 3. The results of Experiment 1. (A) rigidity ratings and (B) motion ratings. (Error bars indicate SEM.)

Acuity of at least $20 / 40$ (Snellen eye chart) was required in the eye that was used throughout the experiment.

Design. The eccentricity of the simulated ellipsoid was the single independent variable examined, with eccentricities of 1.5, 2.0, 2.5, 3.0, and 3.5. Each subject responded to five trials for each shape.

Stimuli. A stimulus simulated an opaque object moving in front of a background plane of 4,400 randomly placed points of light. The motion simulated by the opaque object was constant angular velocity rotation about a fixed vertical axis passing through the center of the ellipsoid. The 4,400 background points were contained within a circle of diameter $23 \mathrm{~cm}(2,657$ plotting positions) and subtended a visual angle of $7^{\circ}$

Since complete rotation of the object would have provided an obvious cue to the correct response, the extent of the object's simulated rotation was $30^{\circ}$ in one direction and $30^{\circ}$ in the opposite direction (at $0^{\circ}$, the major axis was pointed toward the viewer). The change in angular velocity, at the extremes of rotation, was an in- 
stantaneous reversal in direction. The stimulus onset asynchrony (SOA) for all displays was $67 \mathrm{msec}$, and 24 frames $\left(75^{\circ} / \mathrm{sec}\right)$ were needed to make a complete oscillation ( $120^{\circ}$ back and forth).

Apparatus. The stimuli were presented on an IMI 455 vector graphics display scope with $4,096 \times 4,096$ resolution. The subjects viewed the displays through a tube that limited the field of view to $6^{\circ}$. A 0.6 neutral-density filter was inserted into the tube to remove any apparent traces on the CRT. The eye-to-screen distance was approximately $1.78 \mathrm{~m}$.

Both an ellipsoid of revolution sliced in half along its major axis and a clay model were used to demonstrate the definition of cross section and the motion of the object. The subjects provided shape judgments by adjusting a mouse-operated display on a Macintosh Plus that was located immediately adjacent to the viewing tube. The Macintosh displayed a single ellipse with variable eccentricity. A total of 145 ellipses were available, all with minor axis 1 and major axis varying from 1-4 (eccentricities 1-4). Horizontal motion of the mouse was used to scroll through the 145 ellipses, and a double click was used to select the desired ellipse.

Procedure. Each subject participated in a single session that consisted of 30 trials. The 30 trials were divided into two blocks, with the first 5 trials of the first block for practice. The 25 experimental trials were randomly divided between the two blocks. For one block, the ellipse presented on the Macintosh began at its largest extent; for the other block, it began at its smallest extent. The order (large first, small first) was counterbalanced between subjects. There was approximately a 10-sec delay between each trial and a 1-min rest between the blocks.

The subjects were instructed to adjust the ellipse on the Macintosh until it corresponded to the cross section of the simulated object along its longest dimension. The subjects had unlimited time to make their responses since stimuli were oscillated continuously until a response was made.

\section{Results and Discussion}

The subjects' judgments of the eccentricity of the ellipsoids were analyzed by an ANOVA. The factor of shape yielded a significant effect $[F(4,20)=23.23, p<$ $.01]$, with judged eccentricity increasing as simulated eccentricity increased. Post hoc analysis (Tukey-Kramer)

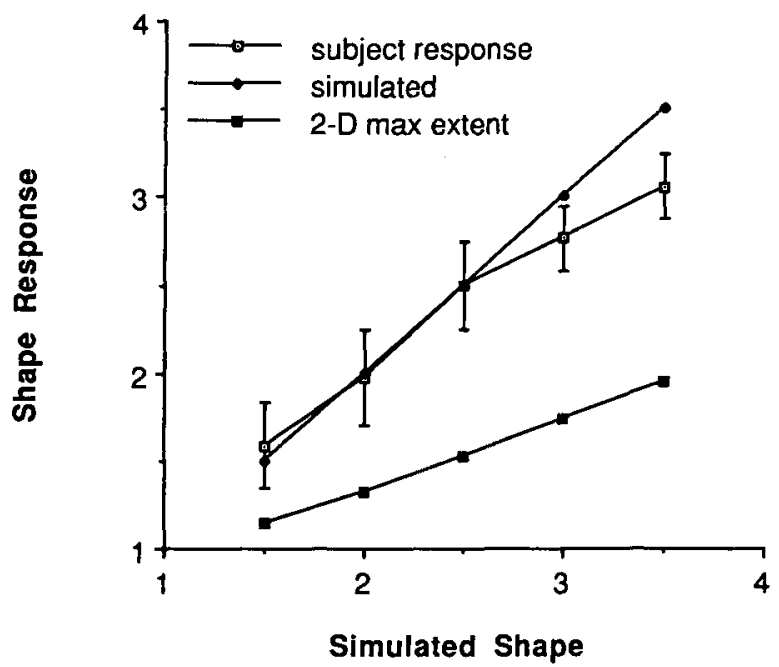

Figure 4. The results of Experiment 2. With subjects' responses, along with the true simulated shape and the maximum twodimensional extent. (Error bars indicate SEM.) showed significant differences for eccentricity 1.5 versus $2.5,3.0$, and $3.5 ; 2.0$ versus 3.0 and 3.5 ; and 3.0 versus $3.5(p<.05)$.

The increase in subjects' shape responses is shown in Figure 4, where it can be contrasted with the increase in simulated shape as well as with the increase in maximum 2-D extent. Maximum 2-D extent is the eccentricity of the largest ellipse of the profile sequence and provides a useful comparison to determine the influence of purely 2-D factors on subjects' responses. It can be seen that the increase in shape responses follows the simulated shape fairly closely, falling off at slightly higher simulated eccentricity. The fact that the pattern of the subjects' responses was more similar to the simulated shape suggests that the subjects were responding to their perceived 3-D shape rather than to a strictly 2-D property of the stimuli. For this reason, it seems unlikely that the results of Experiment 1 were due to a total failure of the perceptual system to organize the zero-elevation stimuli into 3-D form.

\section{EXPERIMENT 3}

Points of correspondence in relative motion are produced when an ellipsoid is elevated, but other display properties also change with this elevation. One such change is that, for equal extents of rotation, the elevated displays have a larger maximum 2-D projected extent. Experiment 3 investigated the perception of 3-D shape over a wide range of elevations to see if the elevation effect was independent of the increase in maximum projected 2-D extent.

\section{Method}

Subjects. Eight naive undergraduate students volunteered for the experiment and received extra course credit for their participation.

Design. Two independent variables were examined: elevation $\left(0^{\circ}\right.$, $5^{\circ}, 15^{\circ}, 30^{\circ}, 50^{\circ}$, and $75^{\circ}$ ) and shape of the simulated object (eccentricities of 2.5 and 3.5). Each subject responded to three trials at each combination of shape and elevation.

Stimuli. The stimuli were generated in a manner similar to that in Experiment 2, with some exceptions. The object's simulated rotation was changed to be asymmetric, with rotation of $20^{\circ}$ in one direction and $40^{\circ}$ in the opposite direction. This change was necessary because otherwise the zero-elevation displays had a greater tendency than did the elevated displays to be incorrectly seen as making complete rotations.

All rotations simulated an angular velocity of $75 \% / \mathrm{sec}$, which was obtained by displaying 24 frames with a SOA of $67 \mathrm{msec}$. The maximum projected extent of the 2.5 shape varied with elevation and was $2.32^{\circ}, 2.33^{\circ}, 2.40^{\circ}, 2.59^{\circ}, 2.91^{\circ}$, and $3.21^{\circ}$ of visual arc, corresponding to the elevation angles $0^{\circ}, 5^{\circ}, 15^{\circ}, 30^{\circ}, 50^{\circ}$, and $75^{\circ}$. Likewise the maximum projected extent of the 3.5 shape was $3.10^{\circ}, 3.11^{\circ}, 3.22^{\circ}, 3.52^{\circ}, 4.02^{\circ}$, and $4.48^{\circ}$ of visual arc, corresponding to the elevation angles $0^{\circ}, 5^{\circ}, 15^{\circ}, 30^{\circ}, 50^{\circ}$, and $75^{\circ}$.

Procedure. The procedure was identical to that of Experiment 2, with the following exceptions: A session consisted of 42 trials, which were divided into two blocks of 24 and 18 trials, with the first 6 trials of the first block for practice. A random ordering of the three repetitions of each combination of shape, angular velocity, and elevation was divided between the two blocks. For each trial, the ellipse appearing on the Macintosh alternated between starting at its greatest and smallest extents. 


\section{Results and Discussion}

The judged ellipsoid eccentricities were analyzed by an ANOVA with factors of elevation and shape. There were two main effects and no significant interactions. The main effect of shape $[F(1,7)=53.4, p<.01]$ showed an increase in judged eccentricity as the simulated eccentricity increased. The main effect of elevation $[F(5,35)=$ $43.8, p<.01]$ showed an increase in judged eccentricity for increasing elevation. Post hoc comparisons showed significant differences for $0^{\circ}$ versus $5^{\circ}, 15^{\circ}, 30^{\circ}, 50^{\circ}$, and $75^{\circ} ; 5^{\circ}$ versus $30^{\circ}, 50^{\circ}$, and $75^{\circ} ; 15^{\circ}$ versus $50^{\circ}$ and $75^{\circ}$; and $30^{\circ}$ versus $50^{\circ}$ and $75^{\circ}(p<.05)$.

Although there was a significant effect for elevation, in order to interpret this effect, it is necessary to look at the role of potential 2-D cues such as the maximum extent of the projected 2-D ellipse. As elevation increases, so does the maximum 2-D extent, shown in Figure 5, which displays plots of both subjects' responses and maximum 2-D extent. For the larger elevations $\left(30^{\circ}, 50^{\circ}\right.$, and $75^{\circ}$ ), subjects' eccentricity judgments can be accounted for by the increasing maximum 2-D extent. However, at smaller elevations such as $0^{\circ}$ and $5^{\circ}, 2-\mathrm{D}$ maximum extent cannot fully account for the increase in reported eccentricity.

The significant increase in judged eccentricity as elevation rose from $0^{\circ}$ to $5^{\circ}$ is important in regard to how points of correspondence arise. Points of correspondence

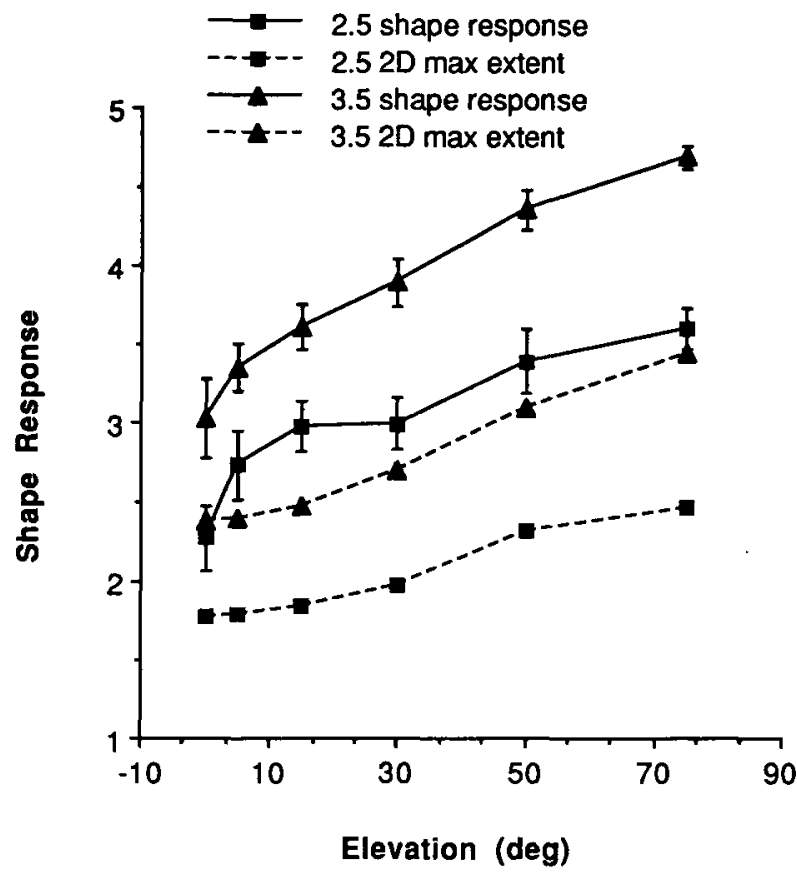

Figure 5. The results of Experiment 3. For both shapes, there is an increase in reported eccentricity with elevation angle. For the high elevations $\left(30^{\circ}-75^{\circ}\right)$, this increase is accounted for by the twodimensional (2-D) maximum extent curves (dashed lines). However, for the small elevations $\left(5^{\circ}-15^{\circ}\right)$, there is little increase in the 2-D maximum extent curve, although there is a significant increase in the judged eccentricity. (Error bars indicate SEM.) appear spontaneously when the ellipsoid is elevated. They are either present in the elevated case or not present in the non-elevated case. Thus, it would be expected that if points of correspondence had an effect, it would show itself abruptly at the slightest elevation.

\section{EXPERIMENT 4}

Taken together, the results of Experiments 1-3 indicate that, compared with their zero-elevation counterparts, elevated ellipsoids are perceived as objects with greater extent in depth. Thus, Experiment 4 explored whether for small elevations this increase in perceived extent could be related to differences in the perceived angular velocity between the elevated and non-elevated displays. This was motivated by the theoretical prediction that the increase in judged eccentricity found with the elevated displays should be associated with a decrease in perceived angular velocity.

\section{Method}

Subjects. Eight naive undergraduate students volunteered for the experiment and received extra course credit for their participation.

Design. Three independent variables were examined: elevation $\left(0^{\circ}\right.$ and $\left.5^{\circ}\right)$, angular velocity $\left(60^{\circ} / \mathrm{sec}, 75^{\circ} / \mathrm{sec}\right.$, and $\left.100^{\circ} / \mathrm{sec}\right)$, and shape of the simulated object (eccentricities of 2.5 and 3.5). Each subject responded to four trials at each combination of these variables.

Stimuli. The stimuli were generated in a manner identical to that used in Experiment 2. The three angular velocities of $60^{\circ} / \mathrm{sec}$, $75^{\circ} / \mathrm{sec}$, and $100^{\circ} / \mathrm{sec}$ were obtained by displaying 30,24 , and 18 frames with an SOA of $67 \mathrm{msec}$.

Apparatus. The apparatus was the same as in Experiment 2, with the addition of a display on the Macintosh Plus for obtaining judgments of angular velocity. This display consisted of a line that rotated at a variable angular velocity. The angular velocity of the line was controlled by the position of a mouse-moving the mouse to the right resulted in a faster angular velocity, and moving the mouse to the left resulted in a slower angular velocity. A double click of the mouse was used to choose an angular velocity. The slowest angular velocity of the rotating line was $36 \% \mathrm{sec}$, and the fastest was $274^{\circ} / \mathrm{sec}$. The length of the line was $2.5 \mathrm{~cm}$, which was equal to twice the minor axis of the family of ellipses used to judge crosssection of the ellipsoid.

Procedure. The procedure was identical to that of Experiment 2, with the following exceptions: Each subject participated in two sessions that consisted of 30 trials each. The 30 trials were divided into two blocks, with the first 6 trials of the first block for practice. Each block contained a random ordering of the 12 combinations of shape, angular velocity, and elevation. The subjects made both the angular velocity and shape judgment for every trial, and the order of making the judgments was constant for a block. For each subject, half the blocks were shape-judgment-first, and half were velocity-judgment-first. At every trial, the angular velocity of the line began at either its maximum or minimum rate. Similarly, the shape of the ellipse began at its maximum or minimum extent. These factors were partially counterbalanced across subjects. There was approximately a $10-\mathrm{sec}$ delay between each trial and a 1-min rest between the blocks. The subjects had a 1-h rest between sessions.

The subjects were instructed to make the two judgments of each stimulus, with the shape judgment being identical to that used in the previous experiments. For the angular-velocity judgment, the subjects were instructed to use the mouse to adjust the angular velocity of the line until it matched the angular velocity of the object 
as seen from a top view. Identical to the previous experiments, the subjects had unlimited time to make their responses, because stimuli were oscillated continuously until both responses were made.

\section{Results and Discussion}

The judged ellipse eccentricities and judged angular velocities were analyzed separately by an ANOVA. The factors in both the ANOVAs were angular velocity, elevation, and shape. For the ANOVA of shape judgments, there were two significant main effects and no significant interactions. The main effect of shape $[F(1,7)=26.9$, $p<.01]$ showed an increase in judged eccentricity as the simulated eccentricity increased. The main effect of elevation $[F(1,7)=8.1, p<.05]$ showed an increase in judged eccentricity for the elevated objects.

The ANOVA of angular velocities yielded two main effects and a significant interaction. The main effect of shape $[F(1,7)=23.0, p<.01]$ showed an increase in judged angular velocity as the simulated eccentricity increased. The main effect of angular velocity $[F(2,14)=$ $29.4, p<.01]$ showed an increase in judged angular velocity as simulated velocity increased. The interaction between shape and elevation $[F(1,7)=7.5, p<.05]$ showed that the reduction in angular velocity for $0^{\circ}$ elevation was significant for the 3.5 ellipsoid but not the 2.5 ellipsoid.

Regarding the velocity judgments, it is interesting to note the overall overestimation of angular velocity and the fact that the larger ellipsoid was perceived as faster than the smaller one. The average simulated angular velocity of the ellipsoids was $78 \% \mathrm{sec}$, whereas the average judged angular velocities were $192 \% \mathrm{sec}$ and $221 \% \mathrm{sec}$ for the 2.5 and 3.5 ellipsoids, respectively. These results are similar to those obtained by Kaiser (1990) for 3-D angularvelocity judgments and Werkhoven (1990) for 2-D rotaryvelocity judgments. Both studies found that smaller objects had to be rotated at a faster angular velocity before they were perceived as rotating at the same angular velocity of a larger object. (Werkhoven reported angularvelocity overestimation by as much as $74 \%$ for an object twice the size of a smaller one.) This suggests that if the rotating line used for angular velocity responses had been longer, the overall degree of overestimation would have been reduced but the larger shape would still have been judged faster.

To check the theoretical prediction that decreased perceived eccentricity should be accompanied by increased perceived angular velocity, it is useful to plot the judgments of eccentricity versus the judgments of angular velocity (Figure 6). It is clear that for each of the six combinations of shape and angular velocity, the zero-elevation ellipsoid was judged to rotate faster than the elevated ellipsoid and had smaller judged eccentricity. This pattern of results (higher angular velocity with smaller shape) agrees with the inverse relationship that was predicted by the computational theories. The finding is also consistent with judgments of shape and extent of rotation obtained from zero-elevation displays (Cortese \& Andersen, 1991).

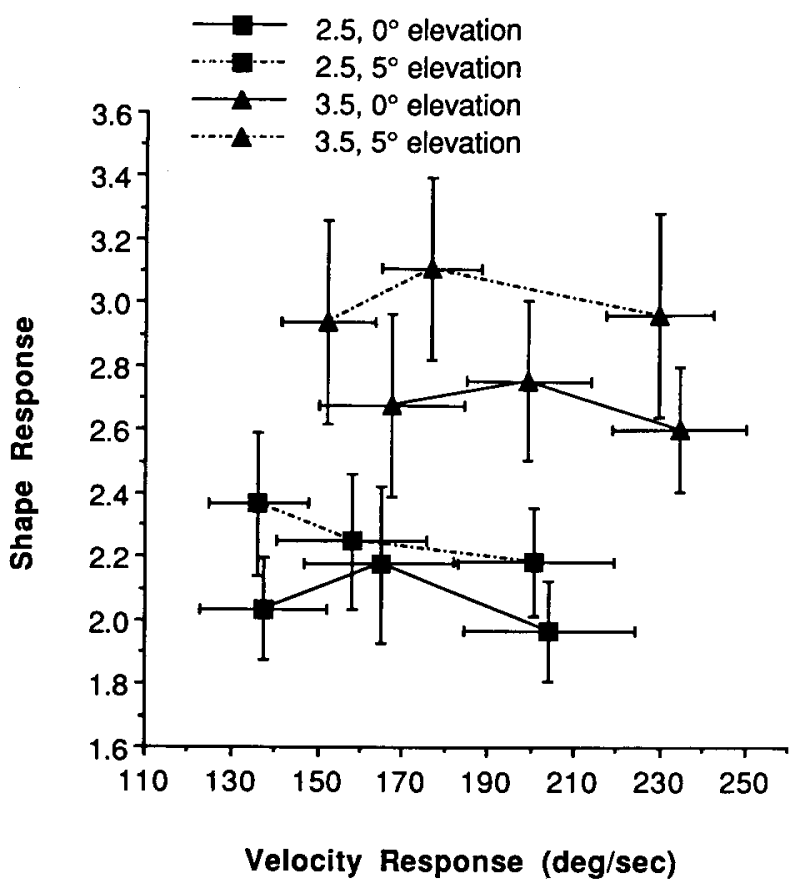

Figure 6. The results of Experiment 4. Subjects' judgments of eccentricity are plotted versus judgments of angular velocity. The zero elevation displays are consistently judged to have a smaller eccentricity and a greater angular velocity. (Error bars indicate SEM.)

\section{Simulations}

It was noted above that, for local measurements of a profile, distance should be inversely proportional to angular velocity (Cipolla \& Blake, 1992; Giblin \& Weiss, 1987; Vaillant \& Faugeras, 1992). Experiment 4 showed just such a relationship, in which increased judgments of eccentricity were accompanied by decreased judgments of angular velocity. This section presents an implementation of an algorithm for the recovery of shape from profiles and applies it to the conditions of Experiment 4 .

The algorithm for shape recovery was based on the theory of Giblin and Weiss (1987). It operated by first measuring the position and image motion of points on the profile, then by using the mean of subjects' judgments of angular velocity, a 3-D shape was recovered.

Given an angular velocity, the shape-recovery algorithm provided a set of points on the recovered surface. To compare these results with subject responses it was necessary to find the ellipsoid of revolution that best fitted the set of points. This was done by fitting the set of recovered points to each of the 145 ellipsoids available to the subjects and choosing the one that minimized the error in the fit to the equation. An example of the best-fitting ellipsoid when the algorithm was inputted with fixed, partial rotations of a zero-elevation ellipsoid is shown in Figure 7. As can be seen, when the algorithm was inputted with higher angular velocities, it produced smaller recovered eccentricities. The simulation results also showed a slight 


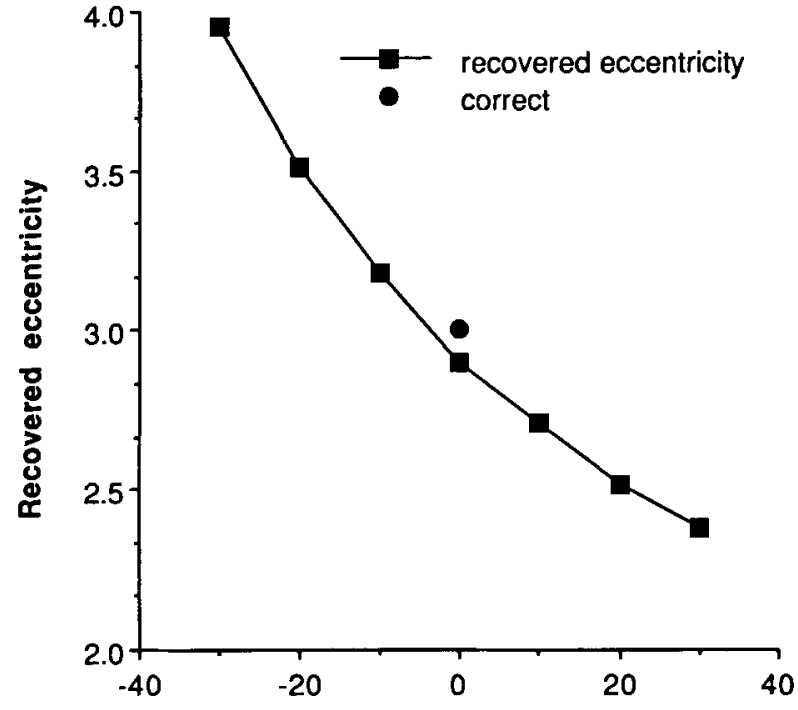

$\%$ Under/overestimate of angular velocity

Figure 7. The recovered eccentricity decreases as the angular velocity supplied to the algorithm increases. These results were obtained for a zero-elevation ellipsoid of revolution (eccentricity 3.0) making incomplete rotations about a vertical axis. The ellipsoid rotated through an extent of $+42^{\circ}$ to $-42^{\circ}$, with the major axis pointing in the view direction at $0^{\circ}$.

underestimation of the eccentricity even when the input angular velocity was correct. However, this is not surprising given that the input to the algorithm consisted of partial rotations that did not completely specify the shape.

To use the shape-recovery algorithm to examine the effect of elevation found in Experiment 4, it was essential to use the subjects' estimates of angular velocity. This was problematic since, as was mentioned before, the subjects showed a general trend to overestimate the angular velocity. This made it necessary to normalize the responses, which was accomplished by assigning the response to the elevated display as veridical (angular velocity 1.0) and calculating ratio of non-elevated to elevated response for the non-elevated display. The assignment of the elevated displays as veridical was arbitrary and restricts the results to pairwise comparisons between elevated and nonelevated conditions. The results are shown in Figure 8, in which it can be seen that all the recovered shapes were smaller for the non-elevated displays.

Comparing the simulation results (Figure 8) with human data from Experiment 4 (Figure 6) demonstrates that the greater angular velocity of the non-elevated displays, when inputted to the algorithm, was sufficient to produce an effect of decreased eccentricity similar to the subjects' shape judgments. And although the calculated elevation effect for the 2.5 ellipsoid was much smaller than that indicated by the subjects' responses, both the results of the subjects and the simulation results showed a larger effect for the 3.5 ellipsoid.

\section{GENERAL DISCUSSION}

Experiment 1 showed that over a wide range of profile displays, the subjects' qualitative judgments of rigidity and motion-in-depth were consistent with the idea that points of correspondence in relative motion were a salient cue to motion-in-depth. Specifically, it was found that there was a considerable difference between responses to single (non-elevated) ellipsoids with no points of correspondence in motion and responses to those (elevated) ellipsoids with points of correspondence in relative motion. The basis of this difference in responses was explored in Experiments 2-4. In Experiment 2, it was shown that the subjects could perceive the profiles of zero-elevation ellipsoids as solid objects moving in depth. In Experiment 3, it was shown that elevated ellipsoids, even for the slightest elevation, were judged to have greater eccentricity than were non-elevated ellipsoids. Since this difference in perceived eccentricity could theoretically have been caused by a difference in perceived angular velocity, Experiment 4 collected responses of both eccentricity and angular velocity. The results of Experiment 4 confirmed this theoretical prediction-the elevated displays that were perceived to have greater eccentricity were also perceived to have smaller angular velocity. Implementing the distance equation of Giblin and Weiss (1987) showed that when the subjects' misjudgments of angular velocity were inputted to the algorithm, a similar pattern of results for shape recovery was produced.

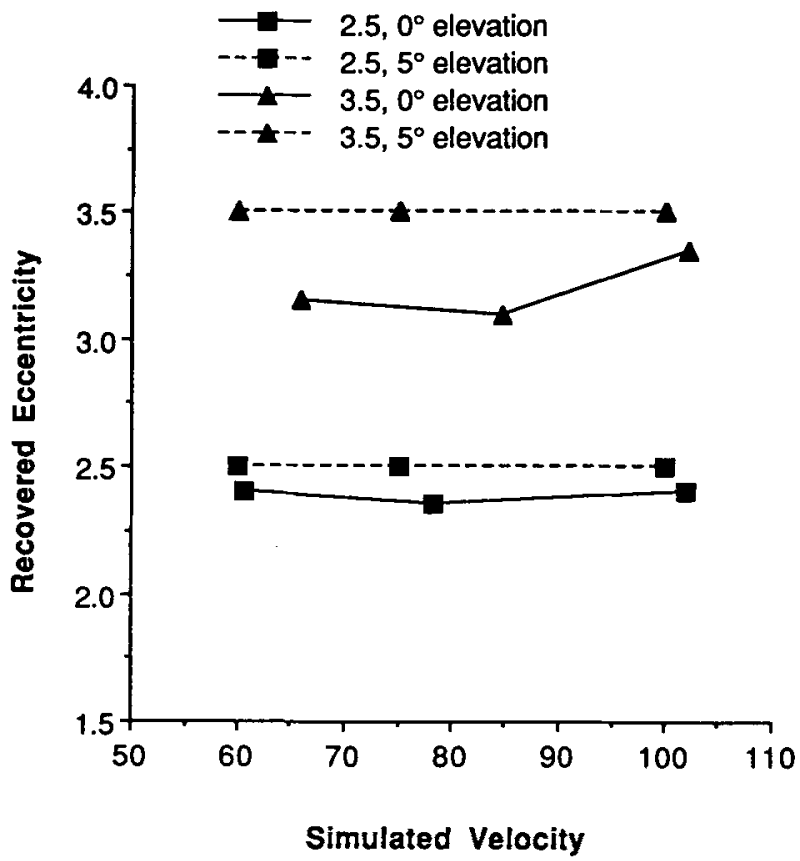

Figure 8. Simulations of Experiment 4. If the elevated responses are considered veridical, it can be seen that the relative difference produced by the algorithm is similar to that produced by subjects. 
A question remains why the zero-elevation ellipsoids were perceived as rotating faster. Since zero-elevation ellipsoids have no points of correspondence in motion, it is difficult to model how angular velocity is measured for these displays. A possible explanation is that the zeroelevation ellipsoids were seen more as a series of views of an ellipse than as the profiles of a solid object. A consequence of this would be a bias to perceive a zeroelevation ellipsoid as rotating faster and as less compelling than a solid object, which is consistent with the results of Experiment 4 and the comments of the subjects during debriefing.

Regarding the perception of profiles derived from a 2-D rather than a 3-D figure, it is important to note that perceiving the profile of an ellipsoid as the projection of an ellipse is in fact correct for a single profile. However, once the ellipsoid begins to move, it is incorrect to attribute the changes in the profile entirely to the motion of an ellipse, because, for the profiles of a solid, the changes are brought on by both the instantaneous motion of the solid and the changing of the set of points that produce the profile. Research by Beusmans (1990) on discriminating between profiles of rotating ellipses and ellipsoids is relevant to this problem. Beusmans found that ellipsoids must be given a fair amount of thickness before they can be discriminated from ellipses. Associated with this increasing thickness (and consequent perception as a solid object) was a decrease of perceived angular velocity. Beusmans postulated that this decrease in perceived angular velocity was due to the profile's no longer being perceived as a rigid space curve.

Combining the results of Kaiser (1990) for the misperception of 3-D angular velocity with the inverse relationship between angular velocity and distance leads to an interesting prediction. Kaiser found that larger objects in 3-D are perceived as rotating faster due to the 2-D bias of their greater 2-D image velocities. The current study suggests that if an object's angular velocity is overestimated, its size will be underestimated. Taken together, these two findings suggest that because of the overestimation of angular velocity, the size of larger objects will be underestimated.

In conclusion, these experiments demonstrated the capability of human subjects to recover shape from the profiles of simple solid objects in motion. They showed results consistent with the theoretical predictions relating perception of shape to perception of angular velocity. In addition, they suggested that the points of correspondence found on profiles are significant in contributing to the perception of motion-in-depth. The close relationship found between theory and experimental results is encouraging, but before it is possible to assert the general validity of current theories, it will be necessary to investigate the effects of more general motions and shapes.

\section{REFERENCES}

Bennett, B., Hoffman, D., Nicola, J., \& Prakash, C. (1989). Structure from two orthographic views of rigid motion. Journal of the $O p$ tical Society of America, 6, 1052-1069.

BEUSMANS, J. M. H. (1990). Visual perception of solid shape from occluding contours (Tech. Rep. 90-40). Irvine: University of California, Department of Information and Computer Science.

BlaKe, A., \& CrPolla, R. (1991). Robust estimation of surface curvature from deformation of apparent contours. Image \& Vision Computing, 9, 107-112.

Cipolla, R., \& Blake, A. (1992). Surface shape from deformation of apparent contours. International Journal of Computer Vision, 9 , 83-112.

Cortese, J. M., \& Andersen, G. J. (1991). Recovery of 3-D shape from deforming contours. Perception \& Psychophysics, 49, 315-327.

Giblin, P. J., Pollick, F. E. , \& RYcroft, J. E. (1993). Recovery of an unknown axis of rotation from the profiles of a rotating surface. Manuscript submitted for publication.

Giblin, P. J., Rycroft, J. E., \& Pollick, F. E. (in press). Moving surfaces. In R. Fisher (Ed.), Mathematics of surfaces $V$ (Proceedings of Institute of Mathematics and its Applications Conference, Edinburgh, September 1992). Oxford: Oxford University Press.

Giblin, P. J., \& SoAREs, M. G. (1988). On the geometry of a surface and its singular profiles. Image \& Vision Computing, 6, 225-234.

GrbLIN, P. J., \& WEIss, R. (1987). Reconstruction of surfaces from profiles. Proceedings of the First International Conference of Computer Vision (pp. 136-144). Los Alamitos, CA: IEEE Computer Society Press.

HofFMAN, D., BENNETT, B. (1985). Inferring the relative 3-D positions of two moving points. Journal of the Optical Society of America A, 75, 350-353.

Huang, T., \& LeE, C. (1989). Motion and structure from orthographic projections. IEEE Transactions on Pattern Analysis \& Machine Intelligence, 11, 536-540.

KAISER, M. K. (1990). Angular velocity discrimination. Perception \& Psychophysics, 47, 149-156.

KoEnderink, J. J., \& VAN DoORN, A. J. (1991). Affine structure from motion. Journal of the Optical Society of America A, 75, 377-385.

Norman, J. F., Todd, J. T., Fuxuda, H. (1992). Perceived 3-D structure from boundary and shadow deformations. Investigative Ophthalmology \& Visual Science, 33, 706.

Poluck, F. E. (1989). Shape perception from dynamic occluding contours. Investigative Ophthalmology \& Visual Science, 30(Suppl.), 264.

Pollick, F. E., Giblin, P. J., Rycroft, L., Wilson, L. L. (1992). Human shape recovery from profiles. Behaviormetrika, 19, 65-79.

Pollick, F. E., Nishida, S., Kolke, Y., \& Kawato, M. (1993). Perceived motion in structure-from-motion: Pointing responses to the axis of rotation. Manuscript submitted for publication.

RIEGER, J. H. (1986). Three-dimensional motion from fixed points of a deforming profile curve. Optics Letters, 11, 123-125.

ToDD, J. T. (1985). Perception of structure from motion: Is projective correspondence of moving elements a necessary condition? Joumal of Experimental Psychology: Human Perception \& Performance, 11, 689-710.

ToDD, J. T., \& Bressan, P. (1990). The perception of 3-dimensional affine structure from minimal apparent motion sequences. Perception \& Psychophysics, 48, 419-430.

VAILlANT, R., \& FAUgeras, O. D. (1992). Using extremal boundaries for 3-D object modeling. IEEE Transactions on Pattern Analysis \& Machine Intelligence, 14, 157-173.

WallaCh, H., \& O'CONNELL, D. N. (1953). The kinetic depth effect. Journal of Experimental Psychology, 45, 205-217.

WERKHOVEN, P. (1990). Visual perception of successive order. Unpublished doctoral dissertation, University of Utrecht, Utrecht, The Netherlands. 

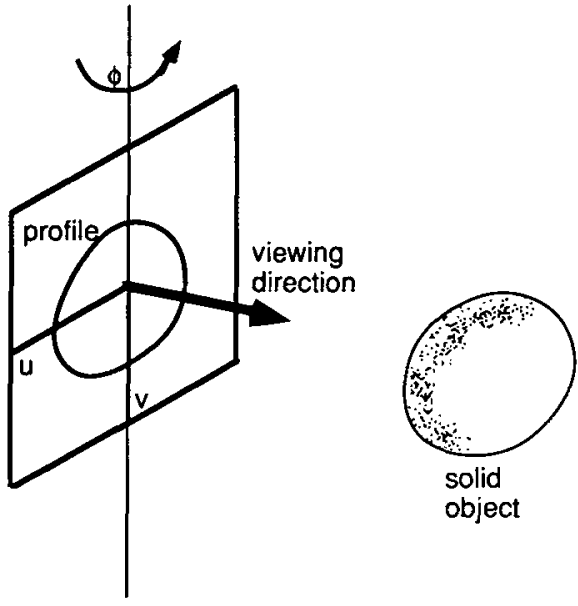

Figure A1. A diagram of the viewing geometry utilized by Giblin and Weiss (1987). Given a viewing direction, the viewplane is defined by the $u, v$ coordinate system. If the viewplane rotates by $\phi$ about the $v$ axis, the corresponding changes in the profile can be measured in the viewplane coordinates, and the surface of the solid object can be reconstructed.

\section{APPENDIX}

This section uses the "distance equation" derived by Giblin and Weiss (1987) to demonstrate the relationship between the motion of an object (angular velocity) and its recovered shape when the axis of rotation is perpendicular to the viewing direction. The result for the general orientation of the viewing direction and axis of rotation is described elsewhere (Cipolla \& Blake, 1992; Giblin et al., in press; Pollick et al., 1992). Figure A1 illustrates the arrangement of coplanar viewing directions used by Giblin and Weiss when the object is fixed and the motion of the viewing direction is known. (Of course, by a transformation, one can obtain the equivalent arrangement in which the viewing direction is fixed and the object rotates about a fixed axis parallel to the viewplane.) Giblin and Weiss found that the relative distance, $d$, from the viewplane to a point on the surface can be expressed as $d=v_{\phi} / v_{u}$ (subscripts denote partial differentiation). The angle $\phi$ gives the orientation of the view- ing direction, and $v$ and $u$ are the coordinates of the viewplane. If one considers the viewing direction to be a function of time, $t$, it is possible to express $v_{\phi}$ as $v_{\phi}=v_{t} / \phi_{t}$, where $\phi_{t}$ is equivalent to the angular velocity of the object and $v_{t}$ is linear velocity in the image plane. Substituting $d v_{u}$ for $v_{\phi}$, one obtains $d=$ $v_{t} t\left(v_{u} \phi_{t}\right)$. Assuming that it is possible to measure the image quantities $v_{t}$ and $v_{u}$, all that is needed to obtain $d$ is $\phi_{t}$. The relationship between $d$ and $\phi_{t}$ is inverse, which implies that if an object is perceived as rotating faster than its actual velocity, it should also be perceived as smaller.

An example of the distance equation for recovering shape when angular velocity is known is shown in Figure A2. For this example, the shape was recovered from an ellipsoid making a full rotation about a vertical axis. It can be seen that although, in general, shape recovery is accurate, there are some regions where portions of the surface are missing. This is due to excluding recovered values at locations where the computation of $d$ showed numerical instability, such as where the tangent was nearly vertical or horizontal. The severity of these instabilities could be ameliorated by more sophisticated numerical techniques of surface interpolation, but it is instructive to look at the behavior of the distance equation by itself.
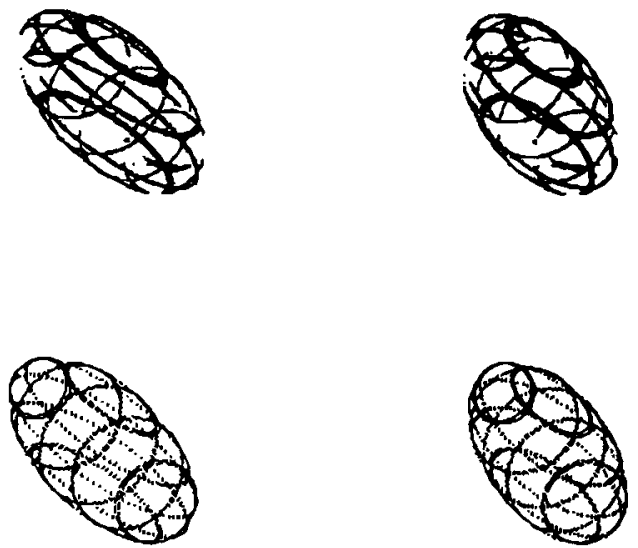

Figure A2. (Bottom) A stereo pair of the skeleton of an ellipsoid input to the algorithm in a series of profiles; (top) the skeleton of the recovered shape.

(Manuscript received June 1, 1992;

revision accepted for publication July 1,1993 .) 